\title{
Visual Network Analysis of Dynamic Metabolic Pathways
}

\author{
Markus Rohrschneider ${ }^{1}$, Alexander Ullrich ${ }^{1}$, Andreas Kerren $^{2}$, \\ Peter F. Stadler ${ }^{1}$, and Gerik Scheuermann ${ }^{1}$ \\ 1 Leipzig University, Department of Computer Science, Germany \\ 2 Linnaeus University, School of Computer Science, Physics and Mathematics (DFM), Sweden
}

\begin{abstract}
We extend our previous work on the exploration of static metabolic networks to evolving, and therefore dynamic, pathways. We apply our visualization software to data from a simulation of early metabolism. Thereby, we show that our technique allows us to test and argue for or against different scenarios for the evolution of metabolic pathways. This supports a profound and efficient analysis of the structure and properties of the generated metabolic networks and its underlying components, while giving the user a vivid impression of the dynamics of the system. The analysis process is inspired by Ben Shneiderman's mantra of information visualization. For the overview, user-defined diagrams give insight into topological changes of the graph as well as changes in the attribute set associated with the participating enzymes, substances and reactions. This way, "interesting features" in time as well as in space can be recognized. A linked view implementation enables the navigation into more detailed layers of perspective for in-depth analysis of individual network configurations.
\end{abstract}

\section{Introduction}

Metabolic networks, the set of chemical compounds and their interactions that constitute life in the most basic sense, are the best studied biological networks. With the plethora of genomic, proteomic and metabolomic data available it becomes possible to study cell behavior. However, to understand the underlying principles of life and gaining further insights about the metabolism of cells for the use in biotechnological applications, e.g., pharmaceutical target prediction or metabolic engineering, we need tools to model and analyze the metabolic processes, pathways, and networks. There exist successful means for the reconstruction of metabolic networks from annotated genomes [1], the analysis of these networks in terms of elementary pathways [2], and description of their behavior with the help of ODE models [3]. Further insight into the development of kinetic models of metabolic networks addressing rate laws of the involved enzymes is provided in [4]. The situation becomes more difficult when we want to explain the evolutionary mechanisms of these systems, i.e., the formation of metabolic pathways or the emergence of complex network properties. Although, several scenarios exist that provide some insight into the evolution of metabolic pathways [5], only few aspects are well understood. Especially, the first steps in early metabolism evade observation by conventional approaches. To this end, Ullrich et al. [6] developed a multi-level computational model to study the transition to life: the evolution of metabolic pathways 
from catalyzed chemical reactions. The simulation approach implements components on different scales in a more realistic manner than has been done so far.

In this work we introduce a plug-in for exploring dynamic graphs extending the existing graph visualization software previously described in [7]. The implementation of the extension was primarily driven by the given data and the requirements stated by the scientists providing it. These include

1. Overview of the complete series of evolving metabolic networks, i.e. involvement of metabolites, reactions and enzymes, and evaluation of key properties, e.g. quantity (concentration) and activity (participation in pathways)

2. Analysis of dynamics in the network's topology and attribute set. Compare networks of different time steps and analyze topology dynamics in more detail.

3. Elementary pathway analysis of selected network generations. Time series analysis of attributes associated with selected node.

For the analysis of the simulation results, an efficient visualization system tailored to suit our needs is of utmost importance. The main function of the software introduced in this article lies in the analysis of metabolic networks in general and studying the evolution and dynamic behavior of metabolism in particular. This is achieved by providing an insightful overview on different scales (e.g., on the metabolite-, pathway-, or network-level) and different angles (e.g., dynamics in topology vs. attribute dynamics) of the vast amount of extracted information. Being able to observe all components (individually or together) for the entire simulation time in one representation gives us a much deeper understanding of the system's dynamics than any statistical analysis or static view can provide. By means of one sample simulation, we show the possibilities of the tool and which potential general insights we can gain.

\section{Related Work}

To deal with large biochemical networks several methods and tools have been developed. Simple approaches try to visualize the complete network on the screen and use zooming and panning for navigation. Examples are common graph drawing or network analysis tools $[9,10]$. Other approaches, such as KGML-ED [11], improve the navigation between single pathways by providing an hierarchical overview and functions to zoom into the top nodes of the hierarchy, or by extending the pathway by connected pathways within the same frame. Our own recent development [7] realized a gridbased visualization approach for metabolic networks supported by a focus\&context view. This view is based on a Table Lens method [12], which provides multiple foci and together with the grid-layout the preservation of the user's mental map, see below. A good overview on open problems and challenges in biological network visualization is provided by the papers $[7,13]$. They provide a comprehensive list of related work, however not focused on the visualization of dynamic biochemical pathways. Oldiges et al. [14] address the specific problem of metabolic network model visualization. However, their article is particularly related to the numerical analysis of dynamic biochemical systems with less emphasis on the visual analysis of the dynamics of the network 
topology. To the best of our knowledge, there is no other visualization tool that focuses on this specific task.

In general, the visualization of dynamic graphs is a well-known area in the graph drawing community [15]. Dynamic graph drawing addresses the problem to layout graphs, which evolve over time by adding and deleting edges and nodes. This results in an additional esthetic criterion known as preserving the mental map [16]. Ad-hoc approaches compute a new layout for the entire graph after each time step using algorithms developed for static graph layout, see for example those presented in the book [9]. In most cases this approach produces layouts which violate the mental map. In our own work, we follow the basic idea of the so-called Foresighted Layout (FL) of dynamic graphs [17]. Given a sequence of $n$ graphs, a global layout is computed, which induces a layout for each of the $n$ graphs. The FL-algorithm is generic in the sense that it takes a static graph drawing algorithm as a parameter. It optimally preserves the mental map. An algorithm for drawing a sequence of graphs online, i.e., where the graph sequence to be laid out is not known in advance, was presented by Frishman and Tal [18].

The general design of our plug-in is based on standard coordinated and multiple view visualization techniques. An excellent starting point for related work of this kind of visualization techniques is the annual conference series on Coordinated \& Multiple Views in Exploratory Visualization (CMV) or the work of Roberts [19]. In our case, the coordination between the different views is mainly done by brushing techniques. The work of Moody et al. [20] focuses on the visualization of dynamic networks in general and the evolution of social networks in particular. The authors state two common approaches: plotting network summary statistics as line graphs over time and examining separate images of the network at each point in time. Our work has been inspired by these two techniques.

\section{The Model}

In this section we introduce a computational model of early metabolism for studying the emergence and evolution of catalyzed chemical reaction networks. The model consists of a graph-based artificial chemistry allowing for realistic kinetic behavior and a proto cell-like entity that inhabits the artificial chemistry and that is exposed to changes (e.g., mutations, source) and selection against other proto cells.

The artificial chemistry of this model is motivated by the chemist's intuition of molecules and chemical reactions. Consequently, molecules are modeled as labeled graphs, with atoms as nodes and bonds as edges. Given this representation, it is easy to see that chemical reactions can be understood as graph transformations, or in computer science terms, as simple graph rewriting rules. Metabolic networks are expanded using a stochastic network generator inspired by Faulon [21]. For simplicity, reaction rates were computed here based on topological indices (Wiener number [22]) of the educt and product molecules of the reactions. The simulation takes two molecules as steady input, namely, the sequential and cyclic form of glucose.

The proto cell contains a simple cyclic genome with several RNA- genes encoding for a particular reaction type (graph rewriting rule) through a sophisticated genotypephenotype mapping [23]. The genome is subject to mutation, deletion, duplication and 
horizontal gene transfer events. Therefore, reactions can occur, change and disappear from the proto cell or even get copied to a neighbor. In each generation, only half of all proto cells is selected and generates an identical copy. There is steady influx of metabolites from the environment and out flux of produced metabolites in way of biomass production. The constitution of either may change during the course of the simulation.

The metabolism of a proto cell is evaluated by analyzing the stoichiometric matrix and fulfilling steady-state and inequality constraints to compute the set of elementary pathways [1].

\section{The Data}

The simulation is run as an adaptive walk over 100 generations. In the analysis of the simulation results, several types of information on different levels are processed. Most importantly, the structure of the metabolic network in form of a bipartite labeled graph is stored in a GraphML [24] file. Metabolites and reactions are the nodes of the graph. An edge leading from a metabolite to a reaction indicates that the respective metabolite is an educt in the reaction, an edge from a reaction to the metabolite node marks the metabolite as product. The labels for reactions are unique identifiers giving insight to their function. The metabolite label is its canonical SMILES string [25], a unique structural representation that is easily readable for chemists. Further, the concentration (number of molecules) for each metabolite is included in the network information.

In addition to the network information, flux information-the set of elementary pathways through the network - is made available to our visualization tool in a simple text file. Extremal nodes are listed. These represent the metabolites transferred into the cell and those that are used as biomass or excreted into the environment, respectively. For each reaction it is noted whether it is present in a particular elementary pathway or not $(0$ or 1$)$.

All types of information are generated for each generation. Since the simulation has several parameters and input options, the data can be very diverse in size and number of files as well as complexity. Here lies also one important merit of this visualization tool. Choosing an "interesting" simulation run for further analysis from the range of possible simulations. The visualization of all levels and generations combined allows an efficient decision process that is of particular importance in a development and testing stage.

\section{The Visualization Framework}

In this chapter we focus on different visualization techniques implemented to support the data analysis process. In this context, mental map preservation is a key requirement for analyzing dynamic networks [16]. Changes in the graph drawing from one network generation to the next should be minimal if the topological changes are small. We achieve the requirement of mental map preservation by following the idea of [17] and create a foresighted layout by constructing the Set Union graph $\hat{G}=(\hat{V}, \hat{E})$ with $\hat{V}=\bigcup_{i=1}^{n} V_{i}$ and $\hat{E}=\bigcup_{i=1}^{n} E_{i}$ where $\left(V_{i}, E_{i}\right)=G_{i}$ are the networks after $i$ generations (see Figure 1). After the preceding cycle removal, we lay out $\hat{G}$ using Sugiyama's method 
for directed acyclic graphs [26,27]. This layout method is suitable for our visualization, because the constructed graph contains very few cycles, and the general direction of fluxes through the network is suggested by the graph drawing, i.e., from top (source) to bottom (sink). To emphasize the importance of extremal nodes-metabolites existing in the cell with no reaction producing them (source metabolites) and metabolites with no reaction consuming them (biomass production) — we connect them to a global source or sink node, i.e, the resulting acyclic graph becomes a so-called st-graph [9]. The set union graph contains the elements of all time steps. Layouting this graph ensures the nodes' positions to remain constant when changing to a different network generation.

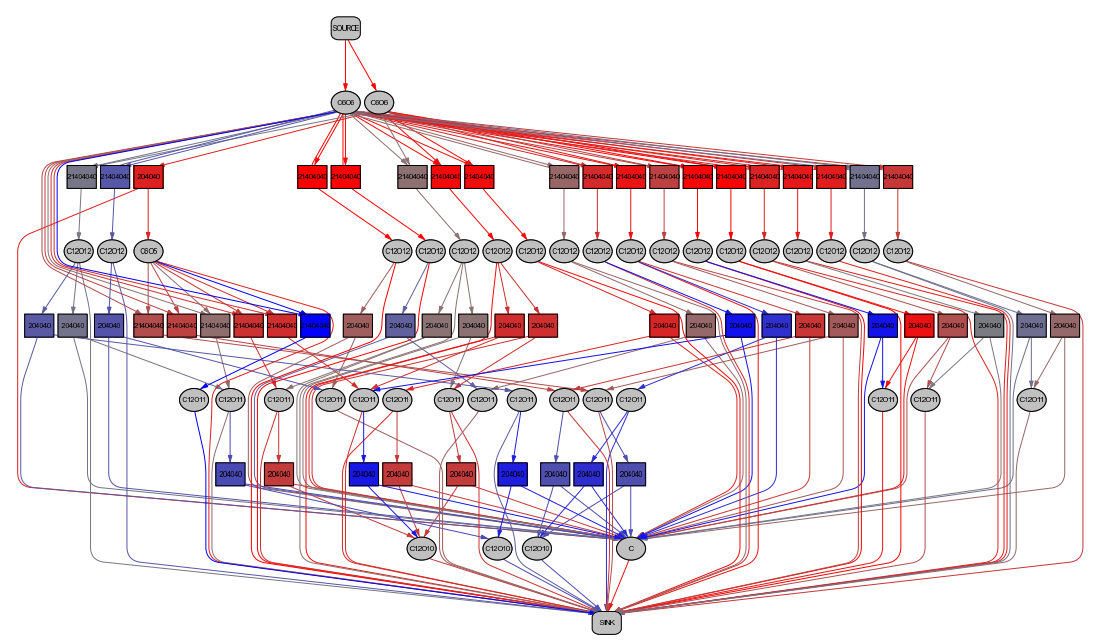

Fig. 1. Set Union Graph laid out using Sugiyama layout algorithm. The reaction nodes (rectangles) are colored according to their first appearance (red: earlier, blue: later).

The three requirements stated in the introduction meet Ben Shneiderman's mantra of information visualization [28]. In the following we describe the visual analysis process based on the scheme "Overview first, zoom and filter, details on demand".

\subsection{Overview}

After construction of the Set Union Graph and associating the flux information with the graph elements, the primary objective of the overview visualization is to give the user a general idea of the network elements-metabolites, reactions and enzymes-involved, their life time, and the development of fundamental attributes associated with the network elements over time (see Figure 2). When presenting the Set Union Graph (a), a given node coloring scheme distinguishes between older and newer nodes. The time of first occurrence of a node in the network determines its color. The node appearing first is red, the node appearing last is blue. Node colors in between are interpolated using the color scale depicted in Figure 7(c), third from left. The user may choose, whether this scheme is applied to reaction nodes, to molecule nodes, or both. 


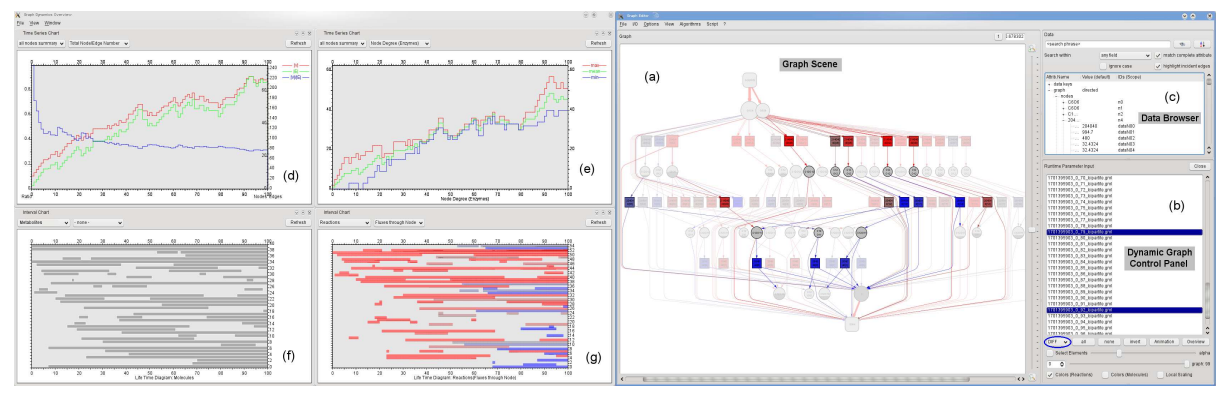

Fig. 2. Graphical User Interface of the Dynamic Graph Analysis plug-in. Overview visualization: Time Series Charts of selected attributes $(\mathrm{d}, \mathrm{e})$ display attribute dynamics over time. Interval Charts (f,g) represent the dynamic topology of the graph in terms of life times of metabolites, enzymes, and reactions. In (g), horizontal bars depicting the nodes' life time have been overlaid with the attribute Fluxes through node. The Graph Scene (a) shows the Set Union Graph with the applied node coloring scheme. As for Zoom and Filter, the user may select different network generations (b) to apply the set operators for filtering elements.

Further insight into the life times of metabolites and reactions give the interval diagrams depicted in Figures 2(e,f). Except for the artificially inserted environment nodes (global source and sink), each row represents a node in the graph. Horizontal bars depict the life time and may be overlaid with additional information, e.g. node degree, fluxes through that node, and concentration for metabolite nodes. In addition to interval diagrams, time series charts $(\mathrm{d}, \mathrm{e})$ summarize selected attributes and display their dynamics over time. The user can again choose the subset of nodes to be taken into account (metabolites, enzymes, or reactions) and the attribute set (node number, node degree, number of elementary fluxes through a node, and concentration values), and combine these time series in any way for comparison.

\subsection{Zoom and Filter}

In this analysis step, the user wants to detect "interesting features" in the overview and select individual networks for further inspection. Interesting in an evolving metabolic network may be periods of stabilities or instabilities in a topological sense-appearance of new reactions or metabolites — as well as in terms of flux behavior-changes of associated attributes.

The straightforward approach is to simply browse the time line. For that purpose, we have implemented a linked view connecting the diagrams of the overview visualization with the dynamic graph in the Graph Scene. The screen shot of the software given in Figure 3 gives an impression on that type of navigation. The user may jump directly to that time point of interest by clicking into any of the displayed diagrams to further inspect the associated network. For each point in time, the current attributes are visualized in the nodes. For metabolites, the concentration values are depicted by the "filling level" of the node. Additionally, the node sizes and edge widths represent the number of elementary pathways these elements participate in. 


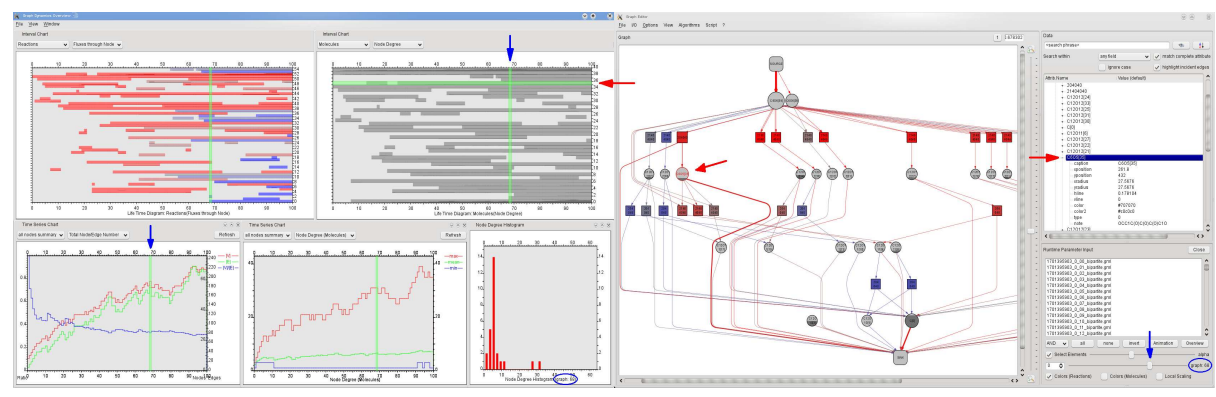

Fig. 3. Linked View realization facilitates browsing different graph snapshots in time. The blue arrows indicate the current position in time, red arrows indicate the selected node in the current generation. These components of the graphical user interface are also sensitive to user input and can be used for navigation. Selecting a node in the Graph Scene (r.h.s.) highlights the associated row in the appropriate interval chart as well as the associated point in time in all charts. The five diagrams given on the 1.h.s. display the following data. Top: Life time diagram of reactions overlaid with the number of pathways through each reaction node. Life time diagram of metabolites overlaid with each node's degree. Bottom: Time series chart giving number of nodes, edges, and nodes-to-edges-ratio. Time series chart of summarized node degree (minimum, maximum, average) over all metabolites. Node degree histogram of the currently displayed graph generation.

For comparing different network generations from a topological point of view, the user may select a number of time steps and apply operators on the node and edge sets of the chosen graph to filter certain elements of the super graph. Set operators include $A N D, O R$, and $D I F F$ for the symmetrical difference between different network snapshots. This is used for detecting subset relations and selecting appearing or disappearing elements (see Figure 2b).

Finally, we have extended the semantic zoom capability in our visualization tool. As the user increases the level of detail, the chemical structures of metabolites is rendered within the associated nodes (see Figure 4).

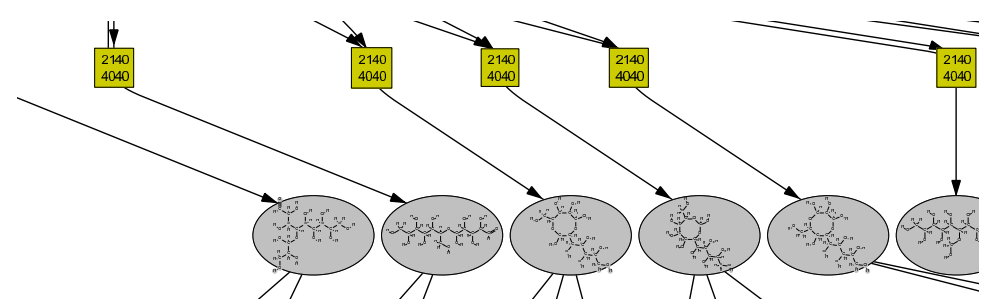

Fig. 4. Semantic Zoom: Below a certain level-of-detail threshold, the chemical structure of the molecule is shown instead of the totals formula. 


\subsection{Detailed View}

In this section, the user takes a closer look on the emergence of individual elementary pathways (fluxes) in a single network evolution step. The aim is to further investigate elements being more or less likely to participate in pathways through the metabolic network and to identify individual elementary pathways. As described in the previous section, the user has identified reactions and metabolites preferred to form pathways as well as key enzymes with high activity. Again, interactivity plays a crucial role in this analysis step. There are two methods of operation: First, the user can select any number of elementary pathways to be highlighted in the Graph Scene displaying the current network generation. Second, the previously identified key elements can be selected in the Graph Scene for highlighting all associated elementary pathways. See the screen shot given in Figure 5. We again implemented set operators on the selected nodes applied for the flux visualization. We found that this is a highly flexible and intuitive way to detect pathways running through all the selected elements $-A N D$ operator, at least one of the selected elements $-O R$, or none of the selected elements $-N O T$.

Concerning the attribute dynamics associated with an enzyme, reaction, or metabolite, we take advantage of the linked view implementation depicted in Figure 3 to display the attribute development of the selected node over time. Selecting a different node instantly updates the displayed time series of the chosen attribute.

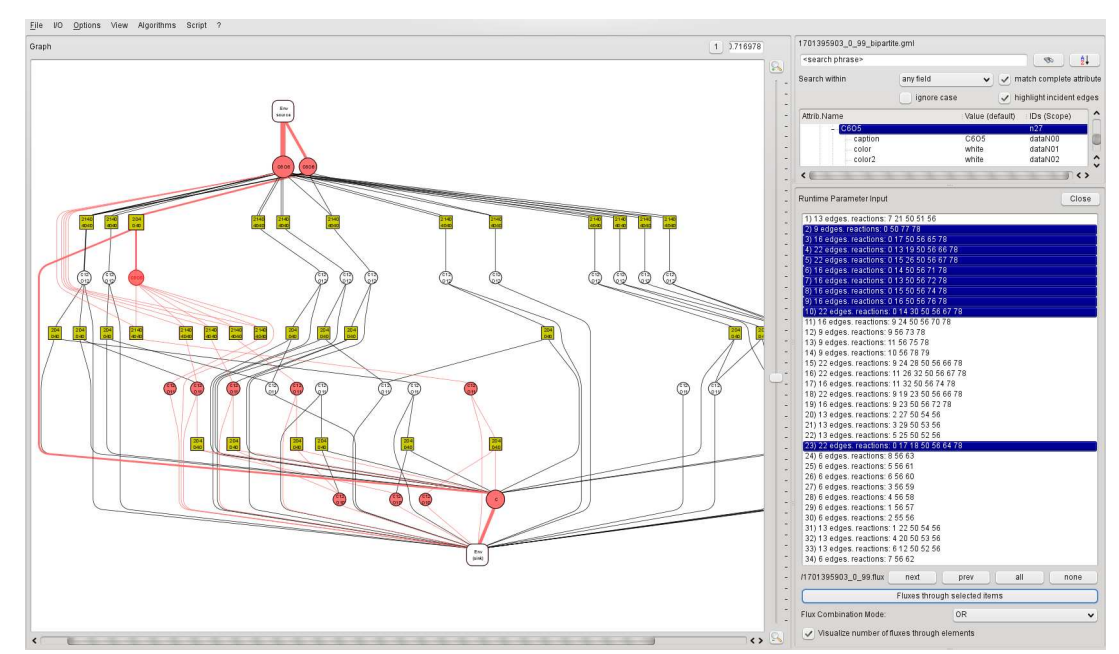

Fig. 5. Details on Demand: Interactive flux analysis for one chosen time step (here: $t=99$ ). Individual elementary pathways can be selected for visualization. All pathways through molecule C6O5 are highlighted. 

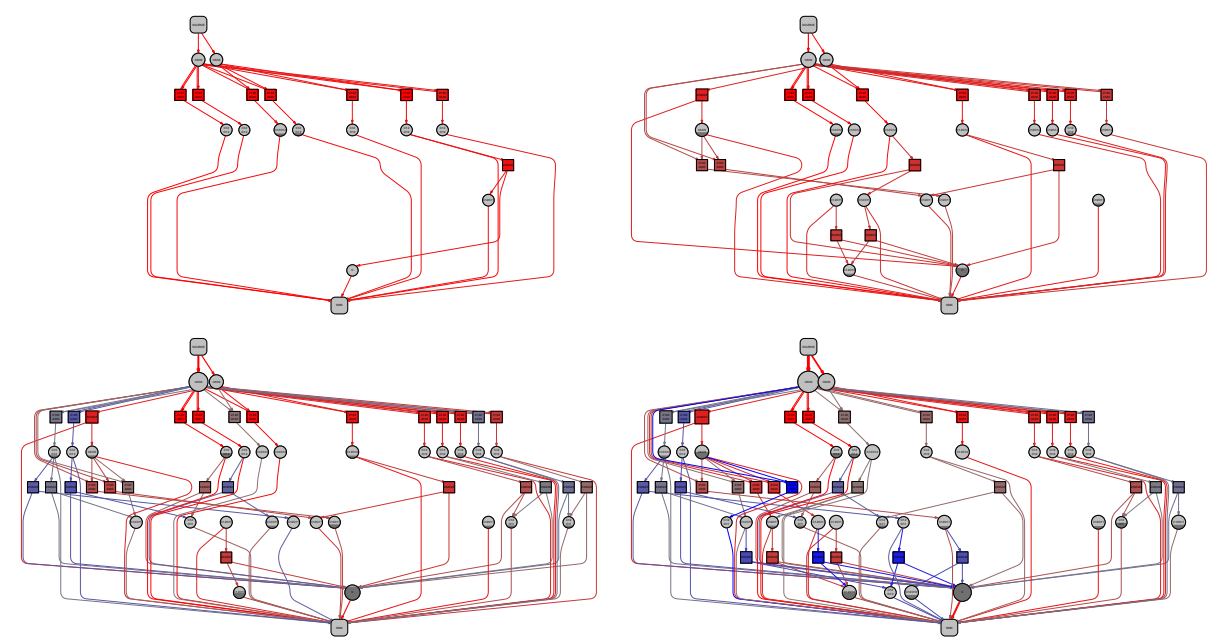

Fig. 6. A series of simulated metabolic networks after 11, 31, 67, and 100 generations. The squares represent reactions, circles represent metabolites. Node size and edge width encode for the number of minimal pathways in which the respective object is involved. Note the dark gray "filling level" of the metabolite nodes depicting the current concentration value.

\section{Results}

In this analysis we wanted to investigate the early steps in the formation and evolution of metabolic pathways and interpret our findings in terms of existing evolutionary scenarios. We will focus on three popular theories, that can be compared nicely to our results. One of the first theories proposed on this matter is backward or retrograde evolution [29], stating that pathways evolved upwards, in the need of finding beneficial substrates due to depletion of metabolites. Contrary, forward evolution [30] suggests the opposite direction of pathways evolution. Due to ever further processing of molecules for energy production, pathways evolve in such a way that ancient enzymes are upstream along the pathway, while younger enzymes are further downstream. The third scenario is the patchwork model [31], which explains the formation of new metabolic pathways through recruiting of enzymes from already existing pathways.

The four snapshots in Figure 6 showing the metabolic network at different points in time are aligned to the union graph over all generations. Thus, we can see that in the first steps the reactions upstream in the network are added. The pathways are formed further in this forward direction. Looking at the last generation, basically all pathways from source to sink follow the forward evolution scenario, with older (red) enzymes being at the top (upstream) and younger (blue) enzymes more at the bottom (downstream). This observation is further established through the interval graph for all chemical reactions in Figure 7. The reactions are here ordered according to their position in the graph. There is a clear trend of older reactions being on the top and younger ones following 


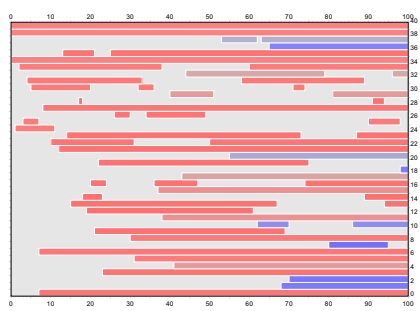

(a)

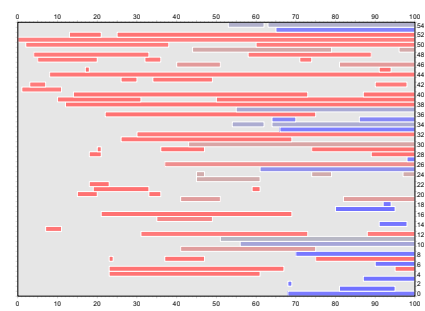

(b)

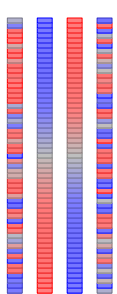

(c)

Fig. 7. Life time diagram of metabolites (a) and reactions (b). Their position in the diagram (yaxis) reflects the associated nodes' positions in the graph layout. Reactions close to the source metabolites are in upper positions, reactions close to the sink metabolites are placed at the bottom. In (c), our scenario (first bar) can be compared to the three evolution models: retrograde evolution, forward evolution, patchwork model.

more downstream. If we compare the colored bars (Figure 7c) showing the enzyme age distribution for our results and the three scenarios mentioned above, the pathway evolution again seems to explain our results best. Therefore, it appears that in the early phase of metabolic evolution, forward evolution is dominant.

We turn now to the evolution of general properties of the metabolic networks from our simulation. The numbers of metabolites and chemical reactions (see Figure 8a) develop with almost the same rate. This indicates that most metabolites are only involved in exactly one reaction. Combining this reasoning with the observation that the maximal node degree of metabolites increases significantly more than their average node degree (see Figure 8b), we can conclude that our metabolic networks evolved one or only a few highly connected metabolites, so called hub-metabolites, and probably has a scale-free node-degree distribution, typical for real-world metabolic networks. Another observation is the steady increase of the average enzyme connectivity while the average metabolite connectivity converges. The explanation for the latter is the high number of metabolites involved in only one reaction. A similar trend will likely arise in more complex stages for enzyme connectivity as well.

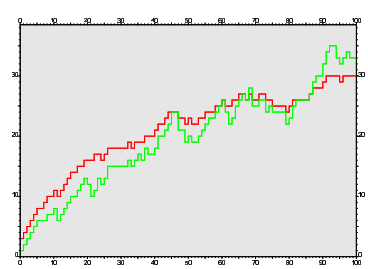

(a)

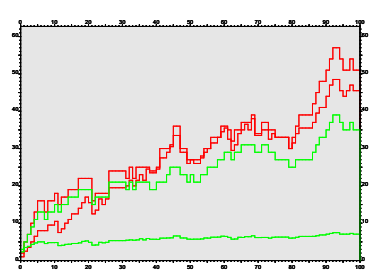

(b)

Fig. 8. Tracking selected attributes over time. (a) Number of metabolites (green) and reaction nodes (red). (b) Node degree (maximum and average) of metabolites (green) and enzyme nodes (red). 


\section{Conclusion and Future Work}

We have presented an extension to our existing graph visualization system to support the exploration and analysis of dynamic metabolic networks. The development process was intensively accompanied by the scientists providing the data and was found to be extremely helpful to understand the underlying mechanisms of metabolic network and biochemical pathway evolution. The visualization could reveal general properties of the considered systems in terms of network topology, but also answered specific questions on the evolution of metabolic networks and the emergence of pathways within the network.

We found that interactivity plays a crucial role in the analysis process. It was successfully implemented using the linked view method for intuitive navigation in time as well as within a selected network configuration. We intend to examine more simulation runs with different parameter configurations to compare the results and to gain a deeper understanding of metabolic network evolution.

For laying out the constructed set union graph, the Sugiyama method has proven to produce the best results. The layout algorithm was a suitable choice due to the fact that the considered network contained only a few number of cycles, and therefore, the observed elementary pathways followed the general direction from top (source nodes) to bottom (sink nodes). The major disadvantage of this layout method is the amount of space required for the drawing. The number of graph elements in the super graph was small enough for a feasible application of this layout algorithm. Datasets with more generations can become very large and too complex for using the applied graph layout. However, there is room for improvement, since many elements in the super graph do not overlap in time and may therefore occupy the same position reducing the total space for the layout.

\section{References}

1. Palsson, B.O.: Systems Biology: Properties of Reconstructed Networks. Cambridge University Press, New York, NY, USA (2006)

2. Gagneur, J., Klamt, S.: Computation of elementary modes: a unifying framework and the new binary approach. BMC Bioinformatics 5 (2004)

3. Yang, K., Ma, W., Liang, H., Ouyang, Q., Tang, C., Lai, L.: Dynamic simulations on the arachidonic acid metabolic network. PLoS Comput Biol 3 (2007) e55

4. Steuer, R., Gross, T., Selbig, J., Blasius, B.: Structural kinetic modeling of metabolic networks. Proc. Natl. Acad. Sci. 103 (2006) 11868-11873

5. Caetano-Anollés, G., Yafremava, L.S., Gee, H., Caetano-Anollés, D., Kim, H.S., Mittenthal, J.E.: The origin and evolution of modern metabolism. The International Journal of Biochemistry \& Cell Biology 41 (2009) 285-297

6. Ullrich, A., Flamm, C.: Functional evolution of ribozyme-catalyzed metabolisms in a graphbased toy-universe. In Istrail, S., ed.: Proc. 6th Int. Conf. on Comp. Methodes in Systems Biology (CSMB). Volume 5307 of Lect. Notes Bioinf. (2008) 28-43

7. Rohrschneider, M., Heine, C., Reichenbach, A., Kerren, A., Scheuermann, G.: A novel gridbased visualization approach for metabolic networks with advanced focus \& context view. In: Proc. 17th Int. Symp. on Graph Drawing (GD 2009). Volume 5849 of LNCS., Springer (2010) 268-279 
8. Kerren, A., Ebert, A., Meyer, J., eds.: Human-Centered Visualization Environments. LNCS Tutorial 4417. Springer (2007)

9. Di Battista, G., Eades, P., Tamassia, R., Tollis, I.G.: Graph Drawing: Algorithms for the Visualization of Graphs. Prentice Hall, New Jersey (1999)

10. Görg, C., Pohl, M., Qeli, E., Xu, K.: Visual Representations. [8] 163-230

11. Klukas, C., Schreiber, F.: Dynamic exploration and editing of KEGG pathway diagrams. Bioinformatics 23 (2007) 344-350

12. Rao, R., Card, S.K.: The table lens: merging graphical and symbolic representations in an interactive focus+context visualization for tabular information. In: CHI '94: Conference companion on Human factors in computing systems, ACM (1994) 222

13. Albrecht, M., Kerren, A., Klein, K., Kohlbacher, O., Mutzel, P., Paul, W., Schreiber, F., Wybrow, M.: On open problems in biological network visualization. In: Proc. International Symposium on Graph Drawing (GD ’09). Volume 5849 of LNCS., Springer (2010) 256-267

14. Oldiges, M., Noack, S., Wahl, A., Qeli, E., Freisleben, B., Wiechert, W.: From enzyme kinetics to metabolic network modeling - visualization tool for enhanced kinetic analysis of biochemical network models. Eng. Life Sci. 6 (2006)

15. Branke, J.: Dynamic graph drawing. In: Drawing graphs: methods and models, London, UK, Springer-Verlag (2001) 228-246

16. Misue, K., Eades, P., Lai, W., Sugiyama, K.: Layout adjustment and the mental map. Journal of Visual Languages and Computing 6 (1995) 183-210

17. Diehl, S., Görg, C., Kerren, A.: Preserving the mental map using foresighted layout. In: Proc. of Joint Eurographics-IEEE TVCG Symp. on Vis., VisSym 2001, Springer (2001) 175-184

18. Frishman, Y., Tal, A.: Online dynamic graph drawing. IEEE TVCG 14 (2008) 727-740

19. Roberts, J.C.: Exploratory visualization with multiple linked views. In MacEachren, A., Kraak, M.J., Dykes, J., eds.: Exploring Geovisualization. Amsterdam: Elseviers (2004)

20. Moody, J., McFarland, D., Bender-deMoll, S.: Dynamic network visualization. American Journal of Sociology 110 (2005)

21. Faulon, J.L., Sault, A.G.: Stochastic generator of chemical structure. 3. Reaction network generation. J. Chem. Inf. Comp. Sci. 41 (2001) 894-908

22. Wiener, H.: Structural determination of paraffin boiling points. Journal of the American Chemical Society 69 (1947) $17-20$

23. Ullrich, A., Flamm, C.: A sequence-to-function map for ribozyme-catalyzed metabolisms. In: ECAL. Volume 5777 of Lect. Notes Comp. Sci. (2009)

24. Brandes, U., Eiglsperger, M., Herman, I., Himsolt, M., Marshall, M.S.: Graphml progress report: Structural layer proposal. In: Proceedings of the 9th International Symposium on Graph Drawing (GD 2001). Volume 2265 of LNCS., Springer (2002) 501-512

25. Weininger, D.: SMILES, a chemical language and information system. 1. introduction to methodology and encoding rules. J. Chem. Inf. Comput. Sci. 28 (1988) 31-36

26. Sugiyama, K., Tagawa, S., Toda, M.: Methods for visual understanding of hierarchical systems. IEEE Trans. Systems, Man, and Cybernetics 11 (1981) 109-125

27. Gansner, E.R., Koutsofios, E., North, S.C., Vo, K.P.: A technique for drawing directed graphs. IEEE Trans. Software Eng. 19 (1993) 214-230

28. Shneiderman, B.: The eyes have it: A task by data type taxonomy for information visualizations. In: VL. (1996) 336-343

29. Horowitz, N.H.: On the evolution of biochemical syntheses. Proc Natl Acad Sci USA 31 (1945) 153-157

30. Cordon, F.: Tratado evolucionista de biologa. Aguilar Ediciones, Madrid, Spain (1990)

31. Jensen, R.A.: Enzyme recruitment in evolution of new function. Annu Rev Microbiol 30 (1976) 409-425 